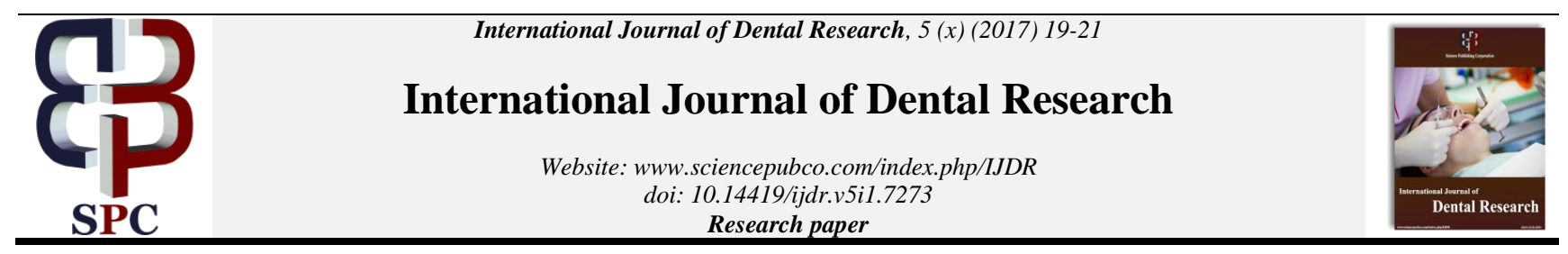

\title{
TUGSE-a curious case series unveiled
}

\author{
Mahima VG' ${ }^{1}$, Karthikeya Patil'², Yesoda Aniyan ${ }^{3}$, Shikha Khare ${ }^{4}$ \\ ${ }^{1}$ Professor,Department if Oral Medicine and Radiology, JSS Dental College and Hospital, Mysuru, Karnataka-570015 \\ ${ }^{2}$ Professor,Department of Oral Medicine and Radiology, JSS Dental College and Hospital, Mysuru,Karnataka-570015 \\ ${ }^{3}$ Post graduate student, Department of Oral Medicine and Radiology, JSS Dental College and Hospital, Mysuru, Karnataka-570015 \\ ${ }^{4}$ Post graduate student, Department of Oral Medicine and Radiology, JSS Dental College and Hospital, Mysuru, Karnataka-570015 \\ *Corresponding author E-mail: patillarthik@gmail.com
}

\begin{abstract}
Traumatic ulcerative granulomas are self-limiting ulcero-proliferative lesions emaluating malignancy clinically, thus causing anxiety for the patient. The etiopathogenesis of these lesions are unclear though trauma is ascertained to be a cause. Riga-Fede disease is considered a variant of the same, manifesting in infants. A clinically varying case series of TUGSE and RFD is reported, and the differential diagnosis discussed here.
\end{abstract}

Keywords: TUGSE; Traumatic Eosinophlic Granuloma with Stromal Eosinophilia; Ulceroproliferative Growth; Riga Fede Disease

\section{Introduction}

Traumatic ulcerative granuloma with stromal eosinophilia (TUGSE) is a chronic benign lesion of the oral mucosa of debatable pathogenesis. It is self-limiting, manifesting as an ulcer with elevated margins. The most common area of occurrence is the tongue. Numerous terminologies have been used to describe TUGSE, including eosinophilic ulcer, eosinophilic granuloma of soft tissue, ulcerative eosinophilic granuloma, eosinophilicumdiutinum and reparative lesion [1]. In infants, however, it is termed, Riga-Fede disease (RFD) [2].

Herein, we report two cases of TUGSE and RFD, affecting the tongue with dissimilar clinical appearances, yet similar histopathological characteristics.

Case report 1

A 54 year old female patient reported to us, with a chief complaint of an asymptomatic ulcer on the tongue, noticed 15 days ago. The anamnesis was non-contributory.On intra oral examination, an ulcero-proliferative growth was noted on the right dorsal surface for the tongue opposing the sharp root stumps, 16 and 17, measuring $1 \times 0.5 \mathrm{~cm}$. The adjacent teeth 46,47 and 48 were missing. The growth was pale pink, circular, sessile and firm in consistency. The superficial ulcer was roughly circular, with pale rolled out edges, regular margins, indurated base and the floor covered with pale yellow necrotic slough. There was no regional lymphadenopathy. The lesion was provisionally diagnosed as TUGSE. The clinical differential diagnosis was squamous-cell carcinoma. On histological examination, after incisional biopsy, hyperplastic stratified squamous parakeratinization of epithelium was apparent with areas of ulceration. The connective tissue consisted of granulomas, epitheliod cells, mixed inflammatory cells, predominantly eosinophils, collagen bundles and fibroblasts. The histological diagnosis was traumatic ulcerative granuloma with stromal eosinophilia.

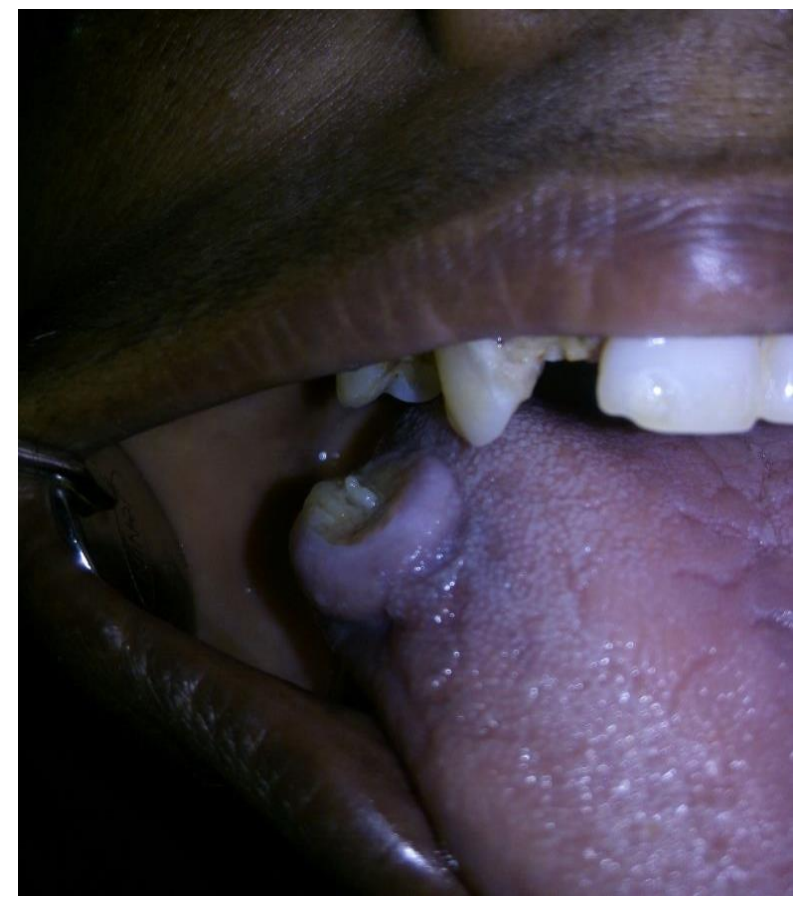

Fig. 1: Ulcero-Proliferative Growth on Right Lateral Border of Tongue.

\section{Case report 2}

A 62-year-old female patient reported with a chief complaint of a painful growth on right lateral border of tongue since 1 day. The patient was a known, diabetic and hypertensive with hypothyroidism. On intra oral examination, a solitary sessile well defined ovalshaped growth was noted on the right lateral border of the tongue coinciding with the sharp cusps of teeth, 46 and 47 , and measuring 1. $2 \times 0.8 \mathrm{~cm}$. The growth was pale, pink and firm with a superficial ulcer. The ulcer had irregular margins, sloping edges and the floor, covered with pale yellow necrotic slough. Regional lymphade- 
nopathy was undetectable. The lesion was provisionally diagnosed as an irritation fibroma with a superficial traumatic ulceration. The clinical differential diagnosis included a fibrosing pyogenic granuloma with superficial ulcer and TUGSE. Excisional biopsy was done. On microscopic examination, the epithelium was stratified squamous parakeratinized and the connective tissue indicated dense chronic inflammatory cells with stromal eosinophilia. The histological impression, concluded TUGSE.

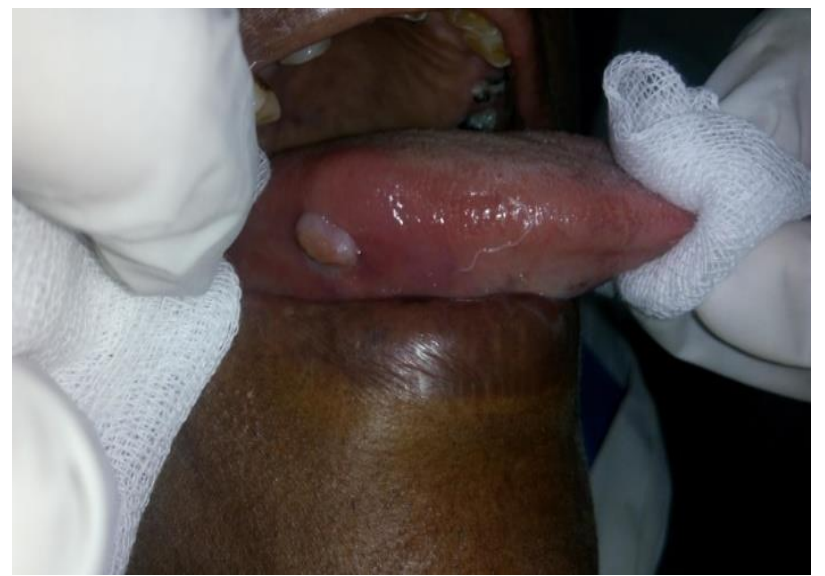

Fig. 2: Growth on Right Lateral Tongue Border.

\section{Case report 3}

A 7 month-old male infant accompanied by his mother reported, with a chief complaint of ulcer of the tongue and associated difficulty in feeding since 1 month. The anamnesis was noncontributory.On intra oral examination, a neonatal tooth that was white and immobile was recorded in the mandibular anterior region. An ulcerated lesion was noted on the midline of anterior ventral surface to the tongue, $8 \mathrm{~mm}$ in diameter. Its margins were regular and edges were pale and rolled-out. The base was indurated and floor, covered with fibro-purulent membrane. It was clinically diagnosed as Riga-Fede disease. Excision and biopsy was not performed considering the age of the subject and the resultant discomfort to tongue.

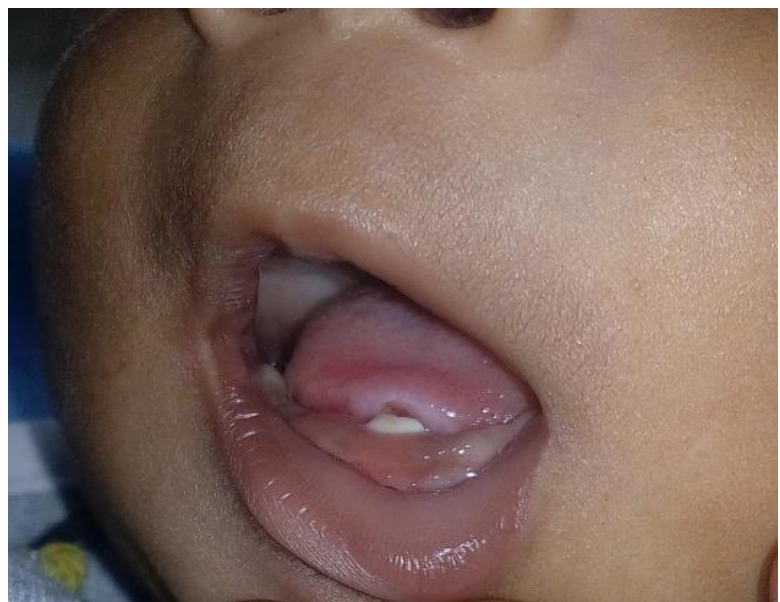

Fig. 3: Ulcerated Lesion on Ventral Anterior Tongue Surface.

\section{Discussion}

TUGSE, delineated as a unique entity, was first described in 1956 in adults by Popoff. In children, Riga and Fede individually described the lesion in 1881 and 1890 respectively and terming it as RFD or Cardarelli aphthae[3].

TUGSE are often solitary lesions varying in size from $0.3 \mathrm{cms}$ to $2.0 \mathrm{cms}$ in diameter. The ulcers appear punched out, with surrounding erythema and keratosis, if present for weeks or months. Regular margins, elevated rolled-out edges, indurated base and floor, comprising yellowish-gray slough is characteristic. Tongue is the common site of occurrence with others being the lip, floor of the mouth, gingiva and palate [2]. Mainly inflicted from crushing injuries in adults, it causes inflammation resulting in myositis which if left untreated, may become deep and penetrating. Occasionally, it presents as a maculae, erythroplakic or leukoplakic lesion. They have rare recurrence except when there is a known cause of repeated trauma. At times, however, it arises in the absence of a traumatic etiology [3]

In children, it is associated with natal or neonatal teeth and may occur in older infants after eruption of the primary lower incisors. According to the classification proposed by Domingues-Cruz et al, RFD can be separated into the precocious type, caused by natal or neonatal teeth, and late disease, with onset after $6-8$ months of age when the lower incisors erupt. This lesion makes it difficult for the infant to suck and feed, putting the baby at risk of nutritional deficiencies. RFD begins as an ulcerated area with prominent raised edges. With repeated trauma, it may progress to an enlarged, fibrous mass with the appearance of an ulcerative granuloma and superficial necrosis. Underlying medical conditions such as Lesch-Nyhan syndrome or familial dysautonomia may present initially as such ulcers.

The duration of TUGSE varies, ranging from multiple weeks to months and sometimes up to a year.Delayed healing of TUGSE lesions has been reported to be associated with the lack of secretion of transforming growth factor TGF-a and TGF-b by eosinophils infiltrating the lesions [4].

The clinical differential diagnosis includes squamous-cell carcinoma, syphilis, tuberculosis, necrotizing sialometaplasia, deep fungal infections, histiocytosis $\mathrm{X}$ and lymphoma. Squamous-cell carcinoma associated ulcers have irregular margins and indurated edges [5]. Syphilitic chancre is usually painless ulcerated indurated nodule and may be differentiated by associated regional lymph node enlargement [6]. However, tuberculous ulcers are asymptomatic, irregular and shallow demonstrating progressive increase in size. A characteristic erythematous halo of depapillated mucosa and indurated edges are also observed [7].Nerotizing sialometaplasia are prevalent on the palate and characterized by a painful rapidly progressive swelling with central ulceration and peripheral erythema [2]. Lymphomas commonly present as a symptomless mass, manifesting a superficial or deep ulceration [9]. Histoplasmosis ulcers present with raised rolled out borders covered by a yellowish gray membrane [8].

Histo-pathologically, TUGSE reveals a dense inflammatory cell infiltrate composed of eosinophils, lymphocytes and large atypical cells with vesicular nuclei and single nucleolus infiltrating the underlying muscle bundles. The histopatholoical differential diagnosis is atypical histiocytic granulomas, angiolymphoid hyperplasia with eosinophilia and Kimura disease [5].

Immunohistochemically, the large cells show variable positivity for CD68, S-100, Factor XIII and vimentin. These cells also show variable positivity for $\mathrm{CD} 30$, a marker expressed commonly by activated $\mathrm{B}$ and $\mathrm{T}$ cells in certain lymphoproliferative disorders, suggesting that TUGSE to be a variant of the same [5],[6].]. In situ hybridization staining conducted by Brasileiro et al was negative for EBER (Epstein-Barr virus-encoded small nuclear RNA) complementary to EBV, EBER1 and EBER2 loci (EBER, PNA probes, DAKO, Denmark),eliminating eliminating the possibility of a virus mediated etio-pathogenesis [10].

Treatment of TUGSE comprises topical or oral analgesics can be applied in painful lesions and surgical excision in persistent ones. Other therapeutic modalities include intralesional and topical steroids, such as a mixture of orabase and corticosteroid ointment (e.g. clobetasol, flucinonide, triamcinolone) or mouthwash, topical antibiotics, curettage, and cryotherapy[2].

Riga-Fede disease management encompasses minimising trauma and extraction is not required. Conservative approach is preferred with smoothing or placement of composite resin over the incisal edges. 


\section{Conclusion}

TUGSE is chronic benign lesions, which feign a malignancy clinically, causing much anxiety. A pediatric variant of it, RFD caused by natal or neonatal teeth is unusual and failure in diagnosis may lead to deformity or mutilation of the tongue, dehydration and inadequate nutrient intake. Hence, an accurate diagnosis and effective yet apt management present the challenge.

\section{Acknowledgements}

The authors acknowledge Dr.Athira Joshy for contributing to this case series.

\section{References}

[1] Abraham Hirshberg,NinetteAmariglio, Sharon Akrish, DMD,RanYahalom, DMD,3 Hanna Rosenbaum, ElimelechOkon, Ilana Kaplan. Traumatic Ulcerative Granuloma with Stromal Eosinophilia: A Reactive Lesion of the Oral Mucosa. Am J ClinPathol $2006 ; 126: 522-529$

Neville Brad,DammDouglas,AllenCarl,ChiAngela.Oral and maxillofacial pathology.First south asianedition.Elsivierhttps://doi.org/10.1309/AFHA406GBT0N2Y64.

[2] Satish K.S. Kumar; Amen Dhyllon; Parish P. Sedghizadeh.Indurated tongue lesion. JADA vol:139 2008

[3] Wei-RenShen, Julia Yu-Fong Chang, Yang-CheWu,Shih-Jung Cheng, Hsin-MingChen , Yi-Ping Wang. Oral traumatic ulcerative granuloma with stromal eosinophilia: A clinicopathological study of 34 cases. Journal of the Formosan Medical Association 2015. https://doi.org/10.1016/j.jfma.2013.09.012.

[4] M.B. Abdel-Naser, F. Tsatsou, S. Hippe, J. Knolle, I. Anagnostopoulos, H. Stein, C.C. Zouboulis. Oral Eosinophilic Ulcer, an Epstein-Barr Virus-Associated CD30+ Lymphoproliferation. Dermatology 2011.

[5] Jessica Swanson, Janna Welch. The Great Imitator Strikes Again Syphilis Presenting as "Tongue Changing Colors". Case Reports in Emergency MedicineVolume 2016

[6] GN Ajay, C Laxmikanth,SK Prashanth .Tuberculous ulcer of tongue with oral complications of oral antituberculosistherapy.Indian journal of Dental research 2006https://doi.org/10.4103/0970-9290.29884.

[7] Krishnan Anitha.Fungal infections of oral mucosa.Indian journal of dental research 2012

[8] Kemp S, Gallagher G, Kabani S, Noonan V,Ohara C: Oral nonHodgkin's lymphoma:review of the literature and World Health Organization classification with reference to 40 cases. Oral Surg Oral Med Oral Pathol Oral RadiolEndod 2008; 105: 194201.https://doi.org/10.1016/j.tripleo.2007.02.019.

[9] Bernardo Ferreira Brasileiro, Daniel Berretta Alves, Bruno Augusto Benevenuto Andrade, Pablo Agustin Vargas, Jorge Esquiche León, OsleiPaes De Almeida. Traumatic ulcerative granuloma with stromal eosinophilia of the palate showing an angiocentric/angiodestructive growth pattern. Contemporary Clinical Dentistry April 2012. 